\title{
Teleneurology: Why it Works for Rural Hospitals
}

\section{Talbot "Mac" McCormick, MD}

The American Academy of Neurology views teleneurology as an effective tool for rapid evaluation of patients in remote locations requiring neurologic care. These underserved locations include geographically isolated rural areas, as well as urban areas with too few available neurology specialists. This article focuses on the problem of stroke worldwide and the penalty imposed on those living in rural areas. Most importantly, the ability to offset those negative factors using teleneurology is illustrated through the real world experience of a rural Oklahoma hospital.

\section{Case Study: INTEGRIS Bass Baptist}

INTEGRIS Bass Baptist Health Center, a rural hospital in Enid, Oklahoma, was unable to provide specialized care to patients without having to transfer them to Oklahoma City or Tulsa - each 100 miles away. The facility searched for a more sophisticated onsite system for treating patients with stroke and other acute neurologic conditions. It was essential that any two-way videoconferencing technology was "always on," readily available when needed, and not used as an emergency department "coat rack"—often the fate of older telemedicine systems.

Through Eagle Hospital Physicians, INTEGRIS Bass Baptist Health Center implemented a state-of-the-art teleneurology program. The positive clinical outcomes are listed in Table 1.

In addition, INTEGRIS Bass was spending over $\$ 300,000$ a year for LocumTenens physician support. Today, INTEGRIS Bass is saving $\$ 200,000$ annually on those costs, and realizing $\$ 100,000$ in income annually that would not have been generated due to reduced patient transfers. Qualitatively, patient acceptance of the technology is positive.

The teleneurology technology at INTEGRIS Bass is always available. Whenever the 
physician from the emergency department calls the program's teleneurologists, they sign on and are immediately interacting in real time with the patient. They conduct a neurologic exam, review the CT scan, talk to the patient and family, and make a recommendation-whether it's administering a thrombolytic drug or other treatment.

\section{Discussion}

Stroke in the US and Worldwide

The findings at INTEGRIS Bass Baptist Hospital have implications for all healthcare providers operating in rural areas. As our nation's population grows older, stroke and its sequelae are increasing. Consider these statistics from the US.

- Every 40 seconds, someone in the United States suffers a stroke

- Strokes are the $5^{\text {th }}$ leading cause of death in the United States

- Strokes are the No. 1 cause of adult disability in the United States

Worldwide, the statistics are equally alarming.

- 2010 prevalence: 33 million, with 16.9 million people having a first stroke

- The leading cause of adult disability

Quantitative neurostereology and stroke neuroimaging research reveal that for every minute during a stroke, a patient loses 1.9 million neurons, 14 billion synapses, and 12 km (7.5 miles) of myelinated fibers. From a practical perspective, for every minute, a patient can be losing pages from the family memory album, losing vocabulary, and losing independence. For every hour of an untreated stroke, a patient loses the brain function equivalent to 3.6 years of normal aging. For a stroke episode of three to six hours, this could mean losing brain function that is typically lost over a period of 10 to 20 years. Teleneurology can help solve this disparity.

\section{The Penalty of Rural Living}

A study by Georgia Health Sciences University underscores the geographic penalty for stroke. It found that for patients who have a stroke in a community served by a hospital with 50 beds or less, the frequency of receiving tissue plasminogen activator (tPA) 
treatment is $12 \%$ what it would be in a community with a 200 -bed hospital. Indeed, chances are good that for people living in a rural area or "underserved acute stroke care" area, they will never receive tPA. The study also found that even most urban and suburban hospitals lack $24 / 7$ acute stroke coverage.

Among those patients who receive treatment, a recent review of the use of tPA revealed that for $23.5 \%$ of patients, front-line hospitals tended to quickly administer tPA to patients with ischemic stroke and then immediately transport them to a more advanced medical center with better stroke treatment facilities. This approach to early treatment is referred to as the "drip and ship" method. However, with this approach to treatment, crude in-hospital mortality (10.9\%) and symptomatic intracranial hemorrhage (5.7\%) were slightly higher compared to patients admitted directly through the "front-door" of the more advanced medical center, and these differences persisted after risk adjustment.

Teleneurology in the rural healthcare setting offers the option to administer IPA at the patient's local hospital. Supporting this protocol, a recent Johns Hopkins Hospital study showed that patients who did not have an intensive care need after the initial hour-long tPA infusion had an extremely low chance of needing any ICU monitoring.

\section{Teleneurology and the Dwindling Supply of Neurologists}

Currently there is an $11 \%$ shortage of neurologists; and this shortage is expected to reach nearly $20 \%$ by 2025 . The uneven geographic spread of neurologists compounds the problem for rural hospitals. For example, neurologist misdistribution ranges from 11.02 neurologists for every 100,000 people in Washington, D.C., to 1.78 neurologists per 100,000 people in Wyoming.

Worldwide, despite the large neurological burden, there is a neurological workforce gap, which is most striking in Africa, where there are 0.03 neurologists per 100,000 people.

Given this scenario, teleneurology marks an important evolution in telemedicine. It 
makes expert care available quickly to even the most remote locations-an important consideration since for treating stroke and other neurological emergencies, every second counts.

In rural hospitals across America, teleneurologists are consulting on the entire spectrum of acute neurologic emergencies, proving effective in diagnosis and treatment of:

- Status epilepticus, coma, and intracranial hemorrhage

- All emergency neurological problems, including utilization of tPA

- $\mathrm{ICH}$ (intracerebral hemorrhage) patients regarding all aspects of medical management, including reversal of anticoagulants

With the assistance of teleneurology, physicians help rural hospitals identify patients requiring transfer to a tertiary care center-such as those who qualify for clot retrieval. They facilitate the handoff by speaking directly to physicians at receiving hospitals. For patients who stay at the rural hospital, the teleneurologist provides effective follow-up care for the remainder of their stay, consulting with patients and providing ongoing support and advice to the local clinical team via two-way videoconferencing.

\section{Part of the Local Team}

Clearly, it is not just the technology that makes or breaks a telemedicine program. An essential best practice is the assignment of telemedicine physicians in pods or small groups for a particular specialty or geography. In this way, they get to know the hospitals to which they are assigned, and the hospital clinical teams get to know them on a first-name basis. The goal is for the remote teleneurology physicians to develop tight working relationships with onsite staff, and for the staff to consider them colleagues. Successful telemedicine physicians have the philosophy that their role is not to dictate, but to augment and support the care by local providers.

\section{Widespread Support}

Telemedicine programs have had time to prove themselves and gain approval from patients, physicians, and hospital administrators. Our surveys find that over $95 \%$ of 
patients report after their very first telemedicine experience that they would recommend it to family or friends. This is in line with others who report that $85 \%$ of patients and $92 \%$ of caregivers as comfortable or very comfortable during telehealth, and another study where $98.3 \%$ of patients were satisfied with their telehealth experience.

We thought elderly patients would be the last holdout in accepting telemedicine, but in many cases they are immediate fans. For example, an elderly patient in a rural Georgia hospital said that all the "cool" technology usually went to Atlanta first, and her community had to wait years for it to "migrate down" to the rural level. She thought it was great that there was finally something that her hospital got before Atlanta.

\section{Three-Fold Return on Investment}

Especially over the last two years, we have witnessed support for teleneurology and other telemedicine programs accelerate rapidly, particularly in rural hospitals. This is the result of hospital leadership recognizing three key benefits: financial return on investment (ROI), community ROI, and patient ROI.

Financial ROI. A typical stroke DRG (diagnosis-related group) reimbursement is $\$ 8,500$. If the patient needs tPA treatment and can stay in the local hospital, the DRG reimbursement level is even higher, typically $\$ 13,000$. This is revenue that hospitals keep when they are equipped to treat patients with stroke and other acute neurological emergencies. And as mentioned by the CFO at INTEGRIS Bass, there are robust cost savings, as well. Return on investment is easy to demonstrate to the Board of Directors.

Community ROI. Beyond financial ROI for hospitals, there is also a community benefit. The rural hospitals we work with are often among the largest employers in their hometowns. By helping them find new care models that contribute to their financial survival, telemedicine programs provide a real service. Nurse practitioners and physician assistants, who are taking on a greater share of the primary care delivered in the United States today, get the support they need. And the support for local hospitalists, emergency department physicians, and other primary care providers is 
invaluable. Local physicians appreciate the access to specialty expertise, and the balance these programs provide their daily—and nightly—work schedule. They report that telemedicine programs have a positive impact on their practices and their quality of life.

Patient ROI. Obviously, patients living in rural communities should not have to continue to suffer geographic penalties for stroke. With teleneurology, this "penalty" is significantly reduced. And when hospitals bring teleneurology or other telemedicine programs to their patients, they are opening the door to $21^{\text {st }}$ century innovations in care-even in the most remote locations.

By enabling patients to stay in their hometown hospital for the care they need, telemedicine programs offer significant stress relief for both patients and their families. It's a benefit not easily quantified, but the physicians in our telemedicine programs see it every day.

Tags: drip and ship, INTEGRIS Bass Baptist, McCormick, neuroimaging, neurology, neurostereology, Oklahoma, patient, rural, satisfaction, stroke, telemedicine, teleneurology, tissue plasminogen activator, tPA,

Talbot "Mac" McCormick, MD is President and CEO, Eagle Hospital Physicians. For more information, contact Eagle Hospital Physicians at info@EagleHospitalPhysicians.com or (678) 441-8529.

Table 1. Results being achieved by best-in-class teleneurologists on our team

- Average response time: 3.5 minutes

- Average diagnosis and treatment time: 21.8 minutes

- Time from emergency department arrival to admission: $40 \%$ decrease

- In hospital average length of stay for stroke patients: 1.2-day decrease 
\#1640. McCormick. Teleneurology.

https://doi.org/10.30953/tmt.v1.72

Page 7 of 7

- Door-to-drip time: Less than 60 minutes, and approaching 40 minutes in some facilities 